\title{
In vivo time domain speckle contrast optical spectroscopy
}

Pagliazzi, M., Vidal-Rosas, E., Konugolu Venkata Sekar, S., Di Sieno, L., Colombo, L., et al.

M. Pagliazzi, E. E. Vidal-Rosas, S. Konugolu Venkata Sekar, L. Di Sieno, L. Colombo, D. Contini, A. Torricelli, A. Pifferi, A. Dalla Mora, J. P. Culver, T. Durduran, "In vivo time domain speckle contrast optical spectroscopy," Proc. SPIE 11074, Diffuse Optical Spectroscopy and Imaging VII, 1107411 (11 July 2019); doi: $10.1117 / 12.2526651$

SPIE. Event: European Conferences on Biomedical Optics, 2019, Munich, Germany 


\title{
In vivo Time Domain Speckle Contrast Optical Spectroscopy
}

M. Pagliazzi ia , E. E. Vidal-Rosas ${ }^{\text {a }}$, S. Konugolu Venkata Sekar ${ }^{\text {b }}$ L. Di Sieno ${ }^{\text {b }}$, L. Colombo ${ }^{\text {, }}$ D. Contini ${ }^{\mathrm{b}}$, A. Torricellib, ${ }^{\mathrm{b}, \mathrm{c}}$, A. Pifferi ${ }^{\mathrm{b}, \mathrm{c}}$, A. Dalla Mora ${ }^{\mathrm{b}}$, J. P. Culver $^{\mathrm{d}},{ }^{\mathrm{b}}$ T. Durduran ${ }^{\mathrm{a}, \mathrm{e}}$

${ }^{a}$ ICFO-Institut de Ciències Fotòniques, The Barcelona Institute of Science and Technology, 08860 Castelldefels, Barcelona, Spain; 'Politecnico di Milano, Dipartimento di Fisica, 20133 Milano, Italy; 'Istituto di Fotonica e Nanotecnologie, Consiglio Nazionale delle Ricerche, 20133 Milano, Italy; ${ }^{\mathrm{d}}$ Department of Biomedical Engineering, Washington University in St. Louis, St. Louis, MO, USA; 'Institució Catalana de Recerca i Estudis Avançats (ICREA), 08015 Barcelona, Spain

\begin{abstract}
We propose a time domain speckle contrast optical spectroscopy (SCOS) system that makes use of a gated detector and pulsed light source to measure the blood flow variations at very short, quasi-null $(<3 \mathrm{~mm})$ source-detector separation. We present the results of a human arm cuff occlusion and a comparison with standard SCOS, highlighting that we can probe deeper into tissue, reduce probe footprint, make efficient use of the signal and decrease cost.
\end{abstract}

Keywords: Time-resolved imaging, speckle imaging, spectroscopy, speckle.

\section{INTRODUCTION}

Non-invasive tissue blood flow monitoring with diffuse correlation spectroscopy (DCS) has been proven important to the assessment of cerebral well being ${ }^{1}$ and brain function ${ }^{2}$. Its widespread use is limited by an elevated cost per channel, which is still several thousand euros to this day. Technical advances moved towards creating a more accessible speckle contrast optical spectroscopy (SCOS) and tomography (SCOT) instrument ${ }^{3}$, which by using detector arrays manages to considerably drop the cost per channel and offers thousands of spatial channels. As with DCS and in general continuous wave techniques, sensitivity to deep tissue (i.e. the brain) in SCOS/SCOT increases with larger source-detector separations, but the contrast and specificity are compromised by the partial volume effect ${ }^{4}$. In time domain (TD) DCS, the state-of-theart instruments have been proven to enhance the deep tissue specificity by employing a pulsed laser source ${ }^{5}$ TD-DCS relies on the fact that in the reflectance geometry, i.e. when the source and the detector are placed on the same side of the tissue, the photons that are detected at later time delays with respect to the pulse emission have statistically traveled deeper into the tissue $\mathrm{e}^{4}$. By iterating the selective detection of these late-arriving photons over the emission of multiple light pulses, one can use TD-DCS to reconstruct the blood flow information reliably ${ }^{4,6}$. However the cost per channel is even higher than for DCS. Each detector channel needs its own timing electronics, i.e. a time-to-digital converter (TDC), or a timecorrelated single-photon counter (TCSPC). Fast-gated single-photon avalanche photodiodes (fgSPADs) are versatile single photon solid state detectors that can be switched on/off in hundreds of picoseconds during every laser pulse period, allowing only the photons impinging on the sensitive area during a window of few nanoseconds to be detected. fgSPADs have been used in NIRS ${ }^{7}$ and recently in TD-DCS ${ }^{8}$ to achieve null separation measurements.

In this contribution, we carry out proof-of-principle analysis of an experimental data from fgSPAD detectors and a custom made titanium-sapphire active mode locked, high coherence laser at quasi-null $(<3 \mathrm{~mm})$ source-detector separation using simplified SCOS analysis which does not require precise timing electronics (TDC or TCSPC). Therefore, as arrays of fgSPAD detectors are being developed, we envision a decrease in the cost per channel, thus paving the road to a widespread use of depth selective and brain specific, high efficiency, non-invasive measurements of blood flow with minimal footprint probes.

\section{EXPERIMENTS AND DISCUSSION}

Coherent light scattered from an ensemble of moving and static scatterers generates a speckle pattern which intensity varies according to the scatterers' motion. Speckle contrast $\kappa^{2}$ is defined ${ }^{3}$ as the variance over the mean square of the timevarying intensity of a speckle over a certain time window. Speckle contrast depends on the autocorrelation

Diffuse Optical Spectroscopy and Imaging VII, edited by Hamid Dehghani, Heidrun Wabnitz, Proc. of SPIE-OSA Vol. 11074, 1107411 · C 2019 SPIE-OSA · CCC code: 1605-7422/19/\$21 · doi: 10.1117/12.2526651 
$g_{I}^{\text {single }}(\tau, s)$ ( $\tau$ is the correlation lag time) of the electric field $(E(t))$ of a single path length $s=v t$, where $t$ is the photon timeof-flight and $v$ its speed in tissue,

$$
g_{1}^{\text {single }}(\tau, s)=\frac{\left\langle E^{*}(t) E(t+\tau)^{*}\right\rangle_{s}}{\left\langle E^{*}(t) E(t)\right\rangle_{s}}=\exp (-\gamma B F i s \tau)
$$

which in turns depends on the blood flow index (BFI). Here, $\gamma$ is a constant that depends on the laser wavelength and tissue's mean refractive index and reduced scattering coefficient, ${ }^{*}$ denotes the complex conjugate of the electric field, and the bracket $\langle\ldots\rangle_{s}$ represents the average over an ensemble of different path lengths of the same length $s$. In a time domain SCOS experiment, carried out by using a pulsed laser source, the speckle contrast $\kappa^{2}$ of only the photons that had time-offlight within a certain gate, i.e. $\left(t_{1}, \infty\right)$, is computed using a fast-time-gated detector in sync with the source pulses, obtaining

$$
\kappa^{2}(T)=\frac{2 \beta}{T} \int_{0}^{T}\left(1-\frac{\tau}{T}\right)\left[\int_{t_{1}}^{\infty} R(t) g_{1}^{\text {single }}(\tau, t) d t\right]^{2} d \tau
$$

where $\beta$ is the SCOS coherence factor, $R(t)$ is the solution of the time resolved diffusion equation for the geometry of interest, and $T$ is the measurement time window. Using a gated detector that selects only the photons arriving after a timeof-flight threshold $t_{l}$ we can get the rid of the preponderant contribution to $\kappa^{2}$ of the short path photons and at the same time investigate the deeper tissue in reflectance geometry, as first suggested for spectroscopy ${ }^{9}$. BFI can be numerically fitted from the measured gated $\kappa^{2}$ using the expressions above.

In our experiments, the source, a titanium-sapphire active mode locked laser operating at $\lambda=785 \mathrm{~nm}$, was split into two branches: one to a photodiode that generated a pulse-sync electronic signal and the other attenuated below skin maximum permissible exposure and launched into a multi-mode graded-index fiber that conveyed light to the probe. In the probe, two single-mode fibers collected the light in reflectance geometry, one at $2.85 \mathrm{~mm}$ distance from the source fiber (fgSPAD connected) and the other at $12 \mathrm{~mm}$ (free running SPAD, MPD, connected). The fgSPAD detector (4 ns gate time, $\mathrm{t}_{1}$ set to $512 \mathrm{ps}$ after the laser peak time, to reject early photons) output was connected to a TCSPC (PH300, Picoquant), along with the pulse-sync, to record the photon arrival times and therefore compute $\kappa^{2}$ by integrating over $\mathrm{T}=\{50,100,200,400,600$, $800,1000,1200,1400,1600,1800,2000,3000,4000,5000,6000,7000,8000,9000,10000\} \mu$ s windows. Much simpler counting electronics could be used but we resorted to TCPSC for practical reasons. The MPD detector was connected to a correlator (Flex05, Correlator.com). In this case, the ungated $\kappa^{2}$ was estimated substituting $g_{I}{ }^{2}$ computed from intensity correlation using the Siegert relation ${ }^{4}$ in equation (2) (replacing the term within square brackets) and setting $t_{1}=0$ to highlight the fact this corresponds to an ungated SCOS experiment, and using the same exposures T as above. An adult healthy human subject (female, 26 years) underwent arm cuff occlusion with a tourniquet placed under the shoulder joint and inflated well above systolic pressure at $\mathrm{t}=180 \mathrm{~s}$ (released at $\mathrm{t}=360 \mathrm{~s}$ ). A soft black foam pad probe, comprising the source fiber and two single mode fibers, was placed above the brachioradialis muscle of the same arm. Superficial tissue thickness in the region was estimated to be $1.3 \mathrm{~cm}$ by using a plicometer.

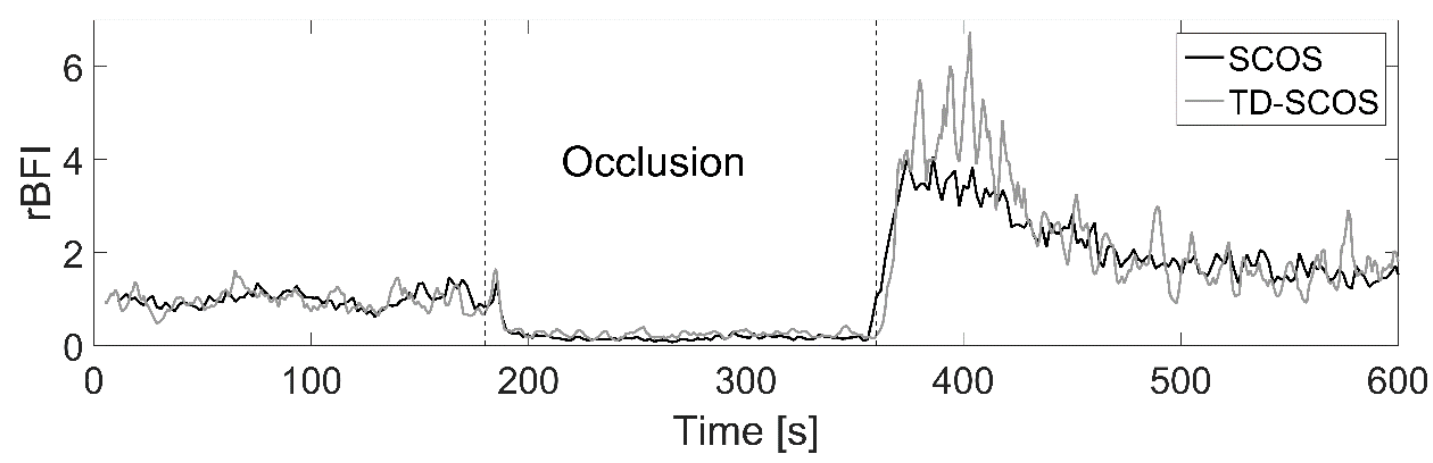

Figure 1. rBFI (normalized to the average BFI of the first $100 \mathrm{~s}$ ) measured with a probe paced above the brachioradialis muscle before ( 0 to $180 \mathrm{~s})$, during (180s to $360 \mathrm{~s})$ and after ( $>360 \mathrm{~s})$ occlusion.

Results are shown in Fig. 1 in terms of relative BFI (rBFI). The graph shows the time series of the rBFI obtained by considering the $\kappa^{2}$ measured with the ungated, $12 \mathrm{~mm}$ separation (SCOS, black line) and the gated, quasi-null separation (TD-SCOS, grey line) before, during and after the arm cuff occlusion. By selecting only the longer time-of-flight photons, the overshoot corresponding to the hyperaemic peak (360 to $430 \mathrm{~s}$ ) is $\sim 33 \%$ higher (albeit slightly noisier) than in the 
ungated case. As in $\mathrm{DCS}^{10}$, this, along with the faster decay captured with TD-SCOS, is also a characteristic of probing a deeper, more metabolically active and reactive muscle tissue.

In conclusion, by using fgSPADSs to compute the gated speckle contrast, the timing electronics used in TD-DCS is not needed, bringing down costs while fully maintaining the enhanced depth selectivity of TD methods for all-optical BFI assessment, which is useful in reflectance non-invasive measurements on the arm or on the head. By rejecting the preponderant unwanted contribution of the photons having small time-of-flight this detection strategy also allows very small source-detector separations to be used. The benefits are smaller footprint probes with enhanced ergonomics and higher density of spatial channels when more detectors are combined in parallel, i.e. in an array or a camera.

\section{ACKNOWLEDGEMENTS}

Fundación Cellex; Instituto de Salud Carlos III (ISCIII) (DTS16/00087); Ministerio de Economía y Competitividad (MINECO) (DPI2015-64358-C2-1-R); "la Caixa" Foundation; "Severo Ochoa" Programme for Centres of Excellence in R\&D (SEV-2015-0522); Agència de Gestió d'Ajuts Universitaris i de Recerca (AGAUR) (2017 SGR 1380); Laserlab Europe IV; Horizon 2020 Framework Programme (H2020) (688303H2020-ICT-2015); Institució CERCA.

\section{REFERENCES}

[1] D. R. Busch, R. Balu, W. B. Baker, W. Guo, L. He, M. Diop, D. Milej, V. Kavuri, O. Amendolia, K. St. Lawrence, A. G. Yodh, and W. A. Kofke, "Detection of Brain Hypoxia Based on Noninvasive Optical Monitoring of Cerebral Blood Flow with Diffuse Correlation Spectroscopy," Neurocrit. Care (2018).

[2] T. Durduran and A. G. Yodh, "Diffuse correlation spectroscopy for non-invasive, micro-vascular cerebral blood flow measurement," Neuroimage 85, 51-63 (2014).

[3] C. P. Valdes, H. M. Varma, A. K. Kristoffersen, T. Dragojevic, J. P. Culver, and T. Durduran, "Speckle contrast optical spectroscopy, a non-invasive, diffuse optical method for measuring microvascular blood flow in tissue," Biomed. Opt. Express 5, 2769 (2014).

[4] F. Martelli, T. Binzoni, A. Pifferi, L. Spinelli, A. Farina, and A. Torricelli, "There's plenty of light at the bottom: statistics of photon penetration depth in random media," Sci. Rep. 6, 27057 (2016).

[5] M. Pagliazzi, S. K. V. Sekar, L. Colombo, E. Martinenghi, J. Minnema, R. Erdmann, D. Contini, A. Dalla Mora, A. Torricelli, A. Pifferi, and T. Durduran, "Time domain diffuse correlation spectroscopy with a high coherence pulsed source: in vivo and phantom results," Biomed. Opt. Express 8, 5311 (2017).

[6] X. Cheng, D. Tamborini, S. A. Carp, O. Shatrovoy, B. Zimmerman, D. Tyulmankov, A. Siegel, M. Blackwell, M. A. Franceschini, and D. A. Boas, "Time domain diffuse correlation spectroscopy: modeling the effects of laser coherence length and instrument response function," Opt. Lett. 43, 2756 (2018).

[7] A. Pifferi, A. Torricelli, L. Spinelli, D. Contini, R. Cubeddu, F. Martelli, G. Zaccanti, A. Tosi, A. Dalla Mora, F. Zappa, and S. Cova, "Time-Resolved Diffuse Reflectance Using Small Source-Detector Separation and Fast SinglePhoton Gating," Phys. Rev. Lett. 100, 138101 (2008).

[8] M. Pagliazzi, S. K. V. Sekar, L. Di Sieno, L. Colombo, T. Durduran, D. Contini, A. Torricelli, A. Pifferi, and A. Dalla Mora, "In vivo time-gated diffuse correlation spectroscopy at quasi-null source-detector separation," Opt. Lett. 43, 2450 (2018).

[9] A. Torricelli, A. Pifferi, L. Spinelli, R. Cubeddu, F. Martelli, S. Del Bianco, and G. Zaccanti, "Time-resolved reflectance at null source-detector separation: improving contrast and resolution in diffuse optical imaging," Phys. Rev. Lett. 95, 78101 (2005).

[10] G. Yu, T. Durduran, G. Lech, C. Zhou, B. Chance, E. R. Mohler, and A. G. Yodh, "Time-dependent blood flow and oxygenation in human skeletal muscles measured with noninvasive near-infrared diffuse optical spectroscopies," J. Biomed. Opt. 10, 24027 (2005). 